\title{
Florence de Caigny, Sénèque le Tragique en France (XVI ${ }^{e}$-XVII ${ }^{e}$ siècles)
}

\section{Michele Mastroianni}

\section{(2) OpenEdition}

\section{Journals}

\section{Edizione digitale}

URL: http://journals.openedition.org/studifrancesi/1127

DOI: 10.4000/studifrancesi. 1127

ISSN: 2421-5856

\section{Editore}

Rosenberg \& Sellier

\section{Edizione cartacea}

Data di pubblicazione: 1 novembre 2014

Paginazione: $586-588$

ISSN: 0039-2944

\section{Notizia bibliografica digitale}

Michele Mastroianni, «Florence de Caigny, Sénèque le Tragique en France (xvi ${ }^{e}$-xvIl ${ }^{e}$ siècles) », Studi

Francesi [Online], 174 (LVIII | III) | 2014, online dal 01 novembre 2014, consultato il 17 septembre 2020. URL : http://journals.openedition.org/studifrancesi/1127; DOI : https://doi.org/10.4000/studifrancesi. 1127

Questo documento è stato generato automaticamente il 17 settembre 2020.

\section{(c) (i) (9)}

Studi Francesi è distribuita con Licenza Creative Commons Attribuzione - Non commerciale - Non opere derivate 4.0 Internazionale. 


\title{
Florence de Caigny, Sénèque le Tragique en France (XVIe-XVII siècles)
}

\author{
Michele Mastroianni
}

\section{NOTIZIA}

FLORENCE DE CAIGNY, Sénèque le Tragique en France (XVI $-\mathrm{XVII}{ }^{\mathrm{e}}$ siècles), Paris, Classiques

Garnier, 2011, pp. 1122.

1 In questo vasto studio sulla ricezione di Seneca in Francia tra Cinque e Seicento, Florence de Caigny offre allo specialista della tragedia del XVI e XVII secolo non solo dati importanti relativi alla ricostruzione del contesto storico-estetico in cui si sviluppa la nuova nozione di tragico cinquecentesca - si tratta, come noto, di una categoria di pensiero, filosofica e letteraria, intrinsecamente al centro della modernità tragica rinascimentale - ma estende il discorso critico a tutto il Seicento, attraverso una molteplicità di informazioni che da un lato consentono di riflettere sui meccanismi teorici, storici e storiografici che regolano l'elaborazione tragica nel periodo indagato e che dall'altro consegnano allo studioso nuovi dati, indispensabili per fare chiarezza su di un asse di elaborazione poetica fondamentale su cui è costruito un genere altrettanto centrale del Cinque e Seicento, in Francia come in Europa.

2 Se il filo conduttore è il senechismo, l'influsso di Seneca, pregnante nella produzione tragica del XVI e XVII secolo francese, si presta - come giustamente mostra Caigny - a una serie di commistioni o sincretismi antichi e moderni che mettono in campo, primo fra tutti, il problema ideologico o teorico della translatio, fortemente dibattuto nel Cinquecento, principalmente rispetto alla questione centrale dell'imitatio. Problema questo del pensiero moderno cinquecentesco, in particolare della rinascita o nascita della tragedia nuova e moderna del xvi secolo, e problema che anticipa e per certi aspetti fonda la grande Querelle des Anciens et des Modernes, attualissima nel Seicento e ancora ricostruibile all'interno delle teorizzazioni concernenti il teatro del Settecento, almeno fino al Père Brumoy (1730). Proprio dal problema dell'elaborazione della 
drammaturgia fra Cinque e Seicento parte questo ricco studio, articolato su tre solide sezioni: 1) La tragédie humaniste et Sénèque: du «poème dramatique» à ses premières «déformations» (1550-1610), pp. 19-346; 2) Les premières contestations d'un modèle installé (1610-1645); pp. 349-682; 3) De la réhabilitation à l'éffacement de Sénèque (1645-1694), pp. 683-1019, sezioni seguite da importanti riflessioni conclusive (pp. 1021-1031) che, con estrema chiarezza, fanno il punto delle ricerche condotte da Caigny. Se da un lato Seneca risulta il grande archetipo della tragedia francese, dagli anni che seguono la pubblicazione della Cléopâtre captive di Jodelle (1553), fino almeno al 1635 - ricordiamo, fra molti, l'influsso sulla Médée di Corneille (1634) e sull'Hippolyte di Guérin de la Pinelière (1635) - anche se, in questo arco temporale, la tragedia lascia spazio alla codificazione di altri generi, come quello della tragicommedia e della pastorale o quello della tragédie en musique e dell'opera, dall'altro, elemento su cui insiste Caigny attraverso una démarche che poggia rigorosamente su dati oggettivi e analisi testuali filologicamente rigorose, risulta evidente che l'autore latino è anche bersaglio di critiche severe, nel Cinque come nel Seicento (sono esemplificative le dichiarazioni del 1555 di Peletier du Mans o quelle del 1639 di La Mesnadière, come rilevanti risultano le considerazioni dell'abbé d'Aubignac che giunge a classificare le tragedie di Seneca «des très mauvais modèles, et il $\mathrm{y}$ en a peu de raisonnables et pas une qui ne soit pleine de fautes et surtout aux Chœurs»). Si direbbe, pertanto, ribadisce l'A., che lo sguardo dei tragediografi sulla produzione tragica senecana si caratterizzi in Francia, tra Cinque e Seicento, per una quasi paradossale tensione estetica che fa contemporaneamente dell'autore latino un modello da imitare e da condannare. Così, partendo dagli studi fondatori sul senechismo di E. Forsyth, K. Böhm, O. Klucke, M. Delcourt-Curves, Fl. Dupont, ecc., Caigny individua un campo di ricerca preciso e la necessità di fare luce sull'influsso di Seneca nel teatro del XVI e XVII secolo francese poiché, come l'A. afferma, «si les études sur le Xvi ${ }^{\mathrm{e}}$ siècle permettent de formuler plusieurs hypothèses - même si elles n'expliquent pas globalement la postérité de l'auteur latin -, le nombre restreint de celles consacrées au XviI ${ }^{e}$ siècle laisse sans réponse la question de la présence sénéquienne à cette époque» (p. 11). Non solo. Il lavoro, ed è certamente questo uno dei grossi pregi del volume, muove dalla necessità di investigare questo periodo - il Cinque, ma soprattutto, come rilevato il Seicento - per assenza di dati critici soddisfacenti, e anche per quella necessaria ricostruzione storica dell'evoluzione di un genere poetico, nella prospettiva appunto parzialmente inedita del senechismo. Si tratta, in particolare, di un grosso studio che offre dati e analisi molto importanti, nonché nuovo materiale, poiché appunto indaga una gamma molto ampia di testi liminari, paratesti e testi tragici che, costantemente, Caigny fa dialogare con il contesto storico dell'epoca, secondo una prospettiva linguistica, stilistica e culturale in genere, quella stessa che anima i grandi débats della Francia del Cinque e Seicento. Del resto Caigny chiarisce fin da subito gli obiettivi delle sue indagini («nous intégrons l'analyse des traductions ou pièces présentées comme telles par la critique jusqu'à ce jour, avec pour objectif de réfléchir sur la nature exacte de ces textes par rapport aux données théoriques des débats sur l'imitation. Ce travail, qui débute avec la Médée de la Pérouse (1556) et se clôt avec la Médée de Longepierre (1694) [...] retrace l'évolution de la réception de l'œuvre dramaturgique sénéquienne au travers de l'étude critique des théoriciens de l'époque, de la définition du rapport des auteurs français avec leur modèle, rapport mis en perspective avec les débats de l'époque sur l'imitation et la tragédie. Enfin, l'analyse du traitement que les auteurs en font sur le plan de l'invention, de la disposition et de l'élocution vise à dégager les transformations de leur modèle: cette troisième étape 
dans notre démarche vise à distinguer l'image de Sénèque véhiculée par la critique tant moderne que contemporaine de l'influence qu'il a réellement exercée sur la tragédie française», p. 14), obiettivi non solo raggiunti, ma verificabili attraverso una lettura attenta di questo lavoro, preziosissimo per il ricercatore. Per esempio, proprio in questa prospettiva si inserisce il primo capitolo, Imitation ou traduction? (1550-1610), pp. 19-118, attraverso il quale Caigny non solo dimostra di leggere con intelligenza i testi, soprattutto le pièces liminaires di tragedie o di trattati importanti del Cinque e Seicento, alla luce del problema della traduzione e dell'imitazione, ma ricostruisce e sintetizza, con estrema chiarezza, i dibattiti e le problematiche teoriche intorno alla traduzione, fornendo un vero e proprio studio nello studio e una sintesi precisa e articolata su quello che, in particolare nella tragedia del Cinquecento, costituisce un elemento nodale della costituzione del tragico rinascimentale (il dibattito, cioè, intorno alla tragedia come laboratorio privilegiato di tecniche traduttive o come luogo di imitazione dei Classici). Partendo dal noto presupposto dell'interesse per il teatro antico greco e latino, l'A. individua nella circolazione - siamo nella prima metà del Cinquecento - di testi come l'Ars poetica di Orazio, il De grammatica di Diomede, il De tragoedia et comedia di Donatus e il Terentius cum quinque Commentis etc., di J. Bade, che raccoglie una serie di commentaires di autori come appunto Orazio e Donatus, una delle cause originarie della riscoperta del pensiero e del teatro di Seneca nel Cinque, ma poi anche nel Seicento. Causa o elemento storiograficamente rilevante che in qualche modo giustifica e informa sull'interesse per l'autore latino, interesse concretamente misurabile se si considerano, per esempio, le cinque edizioni delle tragedie di Seneca che circolano in Francia fra il 1512 e il 1548. Dalla ricostruzione storico-culturale del contesto in cui muove la tragedia rinascimentale, Caigny passa, attraverso l'analisi di una serie di testi e paratesti, alla questione della definizione del genere tragico del Cinquecento francese, per poi ritracciarne lo sviluppo, relativamente al teatro coevo italiano. Attraverso questa prospettiva di indagine, anche comparativa, Caigny conferma il ruolo determinante esercitato nella Francia del XVI secolo dai collèges, che diventano vero e proprio luogo di diffusione dei testi antichi, di tragedie soprattutto. Motivi didattici e formativi spingono autori come Buchanan, Muret e Dorat verso traduzioni latine di tragedie greche, proprio per intenti didattici e morali. Intenti a cui si somma la consapevolezza della 'duttilità' del testo greco antico, facilmente adattabile al nuovo contesto storico-ideologico, da cui muovono - come noto - riattualizzazioni in chiave cristiana delle fabulae antiche. Fra l'altro, come sottolinea l'A., in generale i testi antichi, come nello specifico le tragedie latine «ont permis de comprendre qu'on pouvait composer, sur le modèle des Anciens, des tragédies à sujet historique ou biblique» (p. 45). In questo panorama culturale, le tragedie francesi volte al ricupero dei testi classici, e di Seneca in particolare, non solo circolano nelle scuole, fra gli allievi di Dorat, Muret, Buchanan, ma si prestano anche a un 'processo' di emulazione che si stabilisce fra quegli stessi allievi, in contesti culturali in cui la fruizione del testo si accompagna alla rappresentazione scenica. Tuttavia, l'interesse per il teatro senecano, nella Francia del Cinquecento, non è solo caratterizzato da intenti pedagogici e morali, ma anche da quei contenuti che si prestano a riflessioni di carattere teologico secondo i dibattiti vivacissimi che animano le tensioni dottrinali della seconda metà del Rinascimento, quegli stessi che aprono il campo alle guerre di religione, alla Riforma e Controriforma, almeno fino al Concilio di Trento -, fra i quali è possibile per esempio isolare nozioni lungamente dibattute e di grande rilevo, come quella della predestinazione, del castigo divino, della giustizia di Dio ecc. In ultima analisi, sostiene 
Caigny, «la situation de la France dans la deuxième moitié du xvI ${ }^{e}$ siècle a pesé sur le choix de Sénèque comme source. Dans la période troublée des guerres de religion, ces tragédies latines offraient un vivier important de sujets pouvant illustrer les horreurs contemporaines et les débats idéologiques de l'époque. [...] Pourquoi avoir choisi le théâtre de Sénèque? Les dramaturges n'ont pas uniquement considéré les qualités de l'auteur mais tout ce qu'il représente: une écriture, une philosophie, une représentation des faiblesses de l'homme, une morale, un modèle théâtral qui permet de déployer pathétique et lyrisme» (p. 55). Evidentemente, le questioni in gioco sono numerose e molto complesse, ma l'A. mostra un'attenzione minuziosa per molte di esse, anche se - proprio per l'eterogeneità dei risultati e delle problematiche - non ci è qui possibile riassumerle tutte, ripercorrendo le dense indagini della studiosa. In ogni modo, visto che questa enorme monografia si estende, di fatto, anche a tutto il Seicento tragico, ricordiamo qui brevemente, lasciando poi allo specialista il compito di consultare il materiale offerto da Caigny, i punti essenziali delle indagini. La prima parte - lo abbiamo abbondantemente sottolineato - si costruisce intorno alla elaborazione del tragico cinquecentesco, in rapporto all'influsso senecano, ma anche alla necessità di abbellire e codificare la lingua francese, così come annunciato dalla Pléiade. In effetti, se la tragedia rinascimentale nasce dalla incontestabile emulazione dei testi tragici antichi (greci e latini), il problema o meglio l'esigenza, altrettanto stringente, dell'elocutio fa sì che lo sguardo rivolto agli antichi diventi vettore di modernità tesa, per esempio, verso la creazione di un discorso poetico attento alla costruzione di un linguaggio moderno, appunto, come quello dei poèmes dramatiques e dei poèmes d'amour. La seconda parte della monografia è invece attenta alle variazioni dei dibattiti teorici relativi alla tragedia della prima metà del XVII secolo: «l'essor de genres nouveaux (pastorale, tragi-comédie) qui supplantent la tragédie débouche sur la virulante querelle des règles. Centrée sur le problème de l'application du critère de vraisemblance, elle entraîne la scission des théoriciens qui ne s'accordent pas sur le but à assigner à la tragédie, entre plaire et instruire. Ces réflexions interfèrent dans le rapport aux modèles antiques, qui, s'ils sont toujours considérés comme des références, ne sont pas entièrement recevables. Dans ces circonstances, la restitution de l'esprit de la tragédie antique est accompagnée d'aménagements, la marge de manœuvre laissée aux dramaturges étant réduite en raison du principe de vraisemblance de la fable» (p. 15). In questo senso, precisa sempre Caigny, il problema dell'imitazione diventa - anche nel XVII secolo - problema di elocutio ma anche di inventio, là dove l'esercizio sulle fonti da parte dei tragediografi secenteschi è certamente più complesso rispetto ai modelli antichi e rispetto alle nuove concezioni tragiche che procedono verso drammatizzazioni più propriamente autonome e meno legate a quella classica. La terza sezione, infine, è attenta al ricupero di quelle modalità imitative che segnano il passaggio, relativamente all'esperienza drammatica francese, dalla prima alla seconda metà del XVII secolo, modalità prese in esame alla luce del dibattito sull'imitazione tragica, in particolare degli ultimi cinquant'anni del Seicento. Soprattutto, afferma l'A., le riflessioni teoriche di questo periodo «débouchent sur la définition de règles plus strictes dans la pratique de la traduction, celle-ci sortant du domaine de la création littéraire pour atteindre le statut de genre à part entière» (p. 16). Si tratta, nel caso specifico, di evoluzioni tragiche e, genericamente, di una drammaturgia che si afferma con esiti distinti: da un lato essa tende alla creazione di testi che si caratterizzano ancora per un'attenzione al modello classico, rivisto per così dire attraverso tecniche rielaborative che riconducono, in qualche modo, alla pratica della traduzione, dall'altro 
a pièces che «seront à replacer dans la querelle qui oppose Anciens et Modernes, et qui écarte l'idée d'une restitution de l'esprit de la tragédie antique pour se tourner vers la considération d'un modèle français, défini selon des règles qui lui sont propres» (p. 16). Così, se la tragedia rinascimentale d'ispirazione classica - in questo caso d'ispirazione senecana - può essere certamente considerata anche come genere teso alla creazione di una lingua vernacolare di pari dignità rispetto alle lingue classiche, per parte sua, il modello senecano, nella Francia del Cinque e Seicento, può essere riconosciuto come modello di grande attualità per un genere tragico moderno che passa dalla traduzione all'imitazione e dall'imitazione al libero adattamento, modello attraverso cui la nuova tragedia à la française (nel Seicento in particolare), diventa un luogo di elaborazione dove i prestiti dagli autori antichi assurgono a vero e proprio sostrato culturale per un genere profondamente mutato rispetto al Rinascimento, che tende al soddisfacimento di principi estetici moderni, i quali richiamano il gusto del pubblico cui il teatro è rivolto.

Questo monumentale studio si chiude con una serie di Annexes molto utili per lo studioso, seguite da una ricchissima e aggiornata bibliografia. Non possiamo quindi che insistere ancora sul rigore metodologico, sull'ampiezza dei dati, sulla precisione delle analisi e sull'importanza dei testi presi in esame da Florence de Caigny in un lavoro eccellente che non è solo strumento di lavoro imprescindibile per lo specialista del teatro francese di Cinque e Seicento, ma che è destinato a diventare un testo fondamentale, per non dire poi un classico, della critica letteraria francese sull'espressione tragica del xvi e xvII secolo. 